\title{
Combinatorial Surface Roughness Effects on Osteoclastogenesis and Osteogenesis
}

\author{
Yang Zhang, ${ }^{\dagger}$ S. Elisa Chen, ${ }^{\dagger,}$ Jinlong Shao, ${ }^{\dagger}$ and Jeroen J. J. P. van den Beucken*, ${ }^{\dagger}$ \\ ${ }^{\dagger}$ Department of Biomaterials, Radboudumc, Nijmegen 6525 GA, The Netherlands \\ ${ }^{*}$ Department of Veterinary Medical Science, University of Bologna, Bologna 40126, Italy
}

\section{Supporting Information}

ABSTRACT: Implant surface properties are a key factor in bone responses to metallic bone implants. In view of the emerging evidence on the important role of osteoclasts in bone regeneration, we here studied how surface roughness affects osteoclastic differentiation and to what extent these osteoclasts have stimulatory effects on osteogenic differentiation of osteoprogenitor cells. For this, we induced osteoclasts derived from RAW264.7 cell line and primary mouse macrophages on titanium surfaces with different roughness $\left(R_{\mathrm{a}} \quad 0.02-3.63 \mu \mathrm{m}\right)$ and analyzed osteoclast behavior in terms of cell number, morphology, differentiation, and further anabolic effect on osteoblastic cells. Surfaces with different roughness induced the formation of osteoclasts with distinct phenotypes, based on total osteoclast numbers, morphology, size, cytoskeletal organization, nuclearity, and osteoclastic features. Furthermore, these different osteoclast

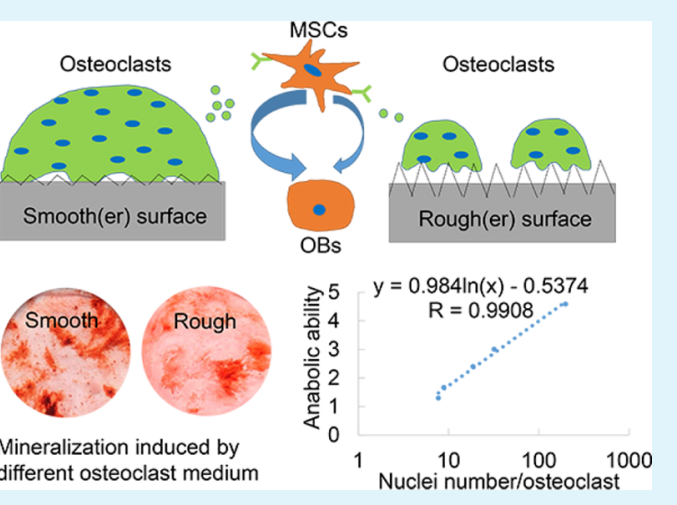
phenotypes displayed differential anabolic effects toward the osteogenic differentiation of osteoblastic cells, for which the clastokine CTHRC1 was identified as a causative factor. Morphologically, osteoclast potency to stimulate osteogenic differentiation of osteoblastic cells was found to logarithmically correlate with the nuclei number per osteoclast. Our results demonstrate the existence of a combinatorial effect of surface roughness, osteoclastogenesis, and osteogenic differentiation. These insights open up a new dimension for designing and producing metallic implants by considering the implant roughness to locally regulate osseointegration through coupling osteoclastogenesis with osteogenesis.

KEYWORDS: surface roughness, osteoclasts, subtype, osteogenic differentiation, coupling of bone resorption and formation

\section{INTRODUCTION}

Metallic biomaterials and devices are widely used in dental, orthopedic, and spinal surgeries to facilitate the replacement and repair of a damaged bone because of their robust mechanical properties and their ability to integrate into the bone (osseointegration). ${ }^{1,2}$ Among these, titanium and its alloys have emerged as the most common bone implant materials and as a model substrate for studying cell and tissue responses to biomaterials because of their clinical relevance, suitable biocompatibility, and the diverse possibilities for surface modifications. ${ }^{3,4}$ Still, clinical work has reported a failure incidence of bone implants up to $14.9 \%$, 5 the majority of which was caused by deficient or poor early bone healing at the bone/implant interface in the early postimplantation period. ${ }^{7,8}$ Consequently, it is of crucial importance to improve the early bone healing of an implant for its long-term performance.

The biomaterial surface properties of an implanted medical device have demonstrated to contribute to the host cellular and tissue responses and play a significant role in determining the overall implant success or failure., ${ }^{9,10}$ Therefore, manipulating the surface physical or chemical properties offers an effective and straightforward strategy to improve the biological performance of implant materials. For titanium implants installed in bone defects in animal studies, multiple studies have shown superior bone-to-implant contact and peri-implant bone formation when the surface roughness (arithmetical mean deviation of the surface profile, $R_{\mathrm{a}}$ ) is between 1 and 1.5 $\mu \mathrm{m} .{ }^{11-13}$ Clinical studies also demonstrated that the osseointegration rate of rough implants was significantly higher than that of machined smooth implants. ${ }^{14-16}$ To elucidate the mechanism responsible for these observations from (pre)clinical studies, previous work has extensively studied how micron and submicron scale roughness contributes to osteoblastic cell attachment, spreading, and differentiation, in which these cells originated from mesenchymal stem cells (MSCs). ${ }^{17-20}$ Remarkably, the effect of other cell types involved in bone remodeling and osseointegration, for example osteoclasts, has to date been largely ignored to reflect the natural interaction of different cell types with a certain surface property. Interestingly, osteoclasts have been observed to appear earlier than osteoblastic cells around bone implants and

Received: July 2, 2018

Accepted: October 1, 2018

Published: October 1, 2018 
seem to initiate the remodeling process to form new bone tissue in the peri-implant region. ${ }^{21-23}$ Furthermore, the preferred roughness of bone implant surfaces, consisting of a combination of micron scale roughness created by sandblasting and submicron scale roughness generated by acid etching, was found to be strikingly similar to osteoclast resorption pit dimensions on bone wafers. ${ }^{24-26}$ All this evidence suggests that the biomaterial surface roughness modulates the behavior of osteoclasts, which further affect the bone formation process.

Osteoclasts are giant multinucleated bone-resorbing cells differentiated from the precursors of the monocyte/macrophage lineage. Polarized osteoclasts form sealing zones, detectable as actin rings and ruffled borders, containing protons and catabolic enzymes such as TRAP, to resorb bone. $^{27,28}$ Osteoclast functions, however, have been widely recognized not to be limited to their ability to resorb bone only. In the context of bone remodeling, osteoclasts also contribute to bone formation by communicating with osteoblastic cells in a process called "coupling of the bone formation to resorption". ${ }^{29}$ In this process, osteoclasts locally promote osteoblastic cell recruitment and osteogenic differentiation through the secretion of coupling factors, known as clastokines, which include sphingosine-1-phosphate (S1P), bone morphogenetic protein 6 (BMP-6), Wnt10b, collagen triple helix repeat containing 1 (CTHRC1), and complement component 3a (C3a). ${ }^{29,30}$ In contrast, sclerostin (Scl) and semaphorin4D (Sema4D), other clastokines secreted by osteoclasts, inhibit osteoblastic cell differentiation. ${ }^{31,32}$ Taken together, these findings indicate that the coupling factors released by osteoclasts play an important role in the local regulation of bone formation by influencing recruitment, osteogenic differentiation, and activity of osteoblastic cells.

Above all, the combined activity of osteoblastic cells and osteoclasts and their bidirectional interactions are relevant for bone remodeling and regeneration. Regarding the chronological order present at the site of bone regeneration and the stimulatory effects of roughness, we hypothesized that the surface roughness influences osteoclastogenesis and the behavior of formed osteoclasts in terms of clastokine secretion. Subsequently, these secreted clastokines then orchestrate osteoblastic cell behavior. To test this hypothesis, we prepared and characterized titanium surfaces with a series of roughness ranging from submicron to micron levels and evaluated their effects on osteoclast behavior (i.e., morphology, cell number, and differentiation) using both the murine RAW264.7 cell line and primary mouse macrophages. To evaluate the coupling function of surface roughness induced osteoclast effects on osteoblastic cell differentiation, the murine MC3T3 osteoprogenitor cell line and primary rat bone marrow MSCs were cultured in the conditioned medium of these osteoclasts, and their osteogenic differentiation was evaluated by mineralization and osteogenic marker analysis and then correlated with potential clastokines and osteoclast-subtype parameters.

\section{MATERIALS AND METHODS}

2.1. Materials and Reagents. Receptor activator for NF- $\kappa \mathrm{B}$ ligand (RANKL) and macrophage colony-stimulating factor (M-CSF) were purchased from Peprotech (Rocky Hill, USA). The Acid Phosphatase Leukocyte Kit and the acid phosphatase activity assay kit were obtained from Sigma (St. Louis, USA). The RNA Isolation Kit was obtained from Qiagen (Venlo, Netherlands). ELF97 dye, Alexa Fluor 568 labeled phalloidin, 4',6-diamidino-2-phenylindole (DAPI), mounting medium, PicoGreen DNA quantification assay kit, TaqMan Reverse Transcription kit, and Fast SYBR Green Master Mix Kit were obtained from Thermo Fisher Scientific (Breda, Netherlands). The osteogenesis quantification kit was obtained from EMD Millipore (Darmstadt, Germany). Minimum essential medium $\alpha$ ( $\alpha$-MEM), fetal bovine serum (FBS), antibiotics, and phosphate-buffered saline (PBS) were obtained from Gibco (Delft, Netherlands). Titanium (Ti) disks $(1.5 \mathrm{~mm}$ in thickness, $12 \mathrm{~mm}$ in diameter; 99.9 wt \% purity) were purchased from Machinefabriek G Janssen (Valkenswaard, Netherlands). Glass coverslips with $12 \mathrm{~mm}$ diameter were obtained from VWR (Renswoude, Netherlands).

2.2. Preparation and Characterization of Titanium (Ti) Surfaces. Ti surfaces with different roughness were prepared through grit blasting with $\mathrm{Al}_{2} \mathrm{O}_{3}$ using different particle sizes $(50 \mu \mathrm{m}$ or 250 $\mu \mathrm{m}$ ) and pressure. To be specific, $50 \mu \mathrm{m}$ of $\mathrm{Al}_{2} \mathrm{O}_{3}$ with 1.2 bar for 10 s, $250 \mu \mathrm{m}$ of $\mathrm{Al}_{2} \mathrm{O}_{3}$ with $1.2 \mathrm{bar}$ for $10 \mathrm{~s}$, and $250 \mu \mathrm{m}$ of $\mathrm{Al}_{2} \mathrm{O}_{3}$ with 3 bar for $10 \mathrm{~s}$ were used to produce low, medium, and high roughness, respectively. Each group of disks was further consecutively washed with $10 \%$ nitric acid, acetone, and ethanol in an ultrasonic bath for 10 $\mathrm{min}$. The disks were then sterilized by autoclaving at $121{ }^{\circ} \mathrm{C}$ for 30 min under 15 psi of pressure. The roughness of the prepared disks was tested with a Universal Surface Tester (UST; Innowep GmbH, Germany). Five disks from each titanium surface were utilized for the test.

2.3. RAW264.7-Derived Osteoclasts. RAW264.7 cell line was obtained from Sigma-Aldrich and cultured in $\alpha$-MEM supplemented with $10 \%$ FBS. Cells were seeded at $2 \times 10^{3} \mathrm{cells} / \mathrm{cm}^{2}$ and passaged until $80 \%$ confluence using a plastic scraper (Greiner Bio-One, Netherlands). For osteoclastic differentiation, $2 \times 10^{3} \mathrm{cells} / \mathrm{cm}^{2}$ RAW264.7 were seeded on the surface of titanium disks and glass slides. The medium was changed to the differentiation medium $(\alpha$ MEM supplemented with $10 \%$ FBS supplemented with $50 \mathrm{ng} / \mathrm{mL}$ of murine sRANK ligand) after $24 \mathrm{~h}$. The medium was then refreshed every 2 days. The cells and the conditioned medium were collected after 4 days of osteoclast induction.

2.4. Mouse Bone Marrow Macrophage Derived Osteoclasts. Mouse bone marrow mononuclear cells were isolated from 6 to 8 week-old male C57Bl/ 6 mice by flushing femurs and tibia with the $\alpha$ MEM medium supplemented with $0.5 \mathrm{mg} / \mathrm{mL}$ of gentamycin and 3 $\mu \mathrm{g} / \mathrm{mL}$ of fungizone. Cells were cultured in $\alpha$-MEM containing $10 \%$ FBS, and the nonadherent cells were collected after $24 \mathrm{~h}$. The cells were then seeded in flasks with $30 \mathrm{ng} / \mathrm{mL}$ of human M-CSF for 2 days. The attached cells were detached and seeded on different surfaces at $2 \times 10^{4}$ cells $/ \mathrm{cm}^{2}$ and cultured in $\alpha$-MEM containing $10 \%$ FBS supplemented with $30 \mathrm{ng} / \mathrm{mL}$ of M-CSF and $50 \mathrm{ng} / \mathrm{mL}$ of murine RANKL for osteoclast induction. The medium was then changed every 2 days. The cells and the conditioned medium were collected after 4 days.

2.5. Characterization of Osteoclast Behavior on Different Roughness. 2.5.1. Cell Morphology. The macrophage morphology on the tested surfaces was examined by scanning electron microscopy (SEM, Jeol SEM6310, Nieuw-Vennep, Netherlands) at indicated time points. Cells were fixed with $2 \%$ glutaraldehyde in cacodylate buffer, dehydrated in a sequential series of ethanol followed by tetramethysilane, coated with gold, and then observed using SEM.

2.5.2. TRAP Staining. Osteoclasts cultured on different surfaces were fixed with $4 \%$ paraformaldehyde (PFA) for $10 \mathrm{~min}$ and stained using the Acid Phosphatase Leukocyte Kit per the manufacturer's instructions. Briefly, $10 \mathrm{~mL}$ of acid phosphatase solution consisting of $9 \mathrm{~mL}$ of prewarmed Milli-Q $400 \mu \mathrm{L}$ of acetate solution, $100 \mu \mathrm{L}$ of naphthol AS-BI phosphoric acid, $200 \mu \mathrm{L}$ of tartrate solution, and 200 $\mu \mathrm{L}$ of diazotized Fast Garnet GBC were prepared, and cells were stained with this solution for $20 \mathrm{~min}$ at $37^{\circ} \mathrm{C}$. The TRAP-positive multinucleated cells on these surfaces were observed using a light microscope (Leica, Germany).

2.5.3. DNA Content. The cell number on different surfaces was assessed using the PicoGreen DNA quantification assay kit. Cell layers were washed twice with PBS, after which $1 \mathrm{~mL}$ of Milli- $\mathrm{Q}$ was added. Following two freeze-thaw cycles, samples were aspirated several times and used for DNA quantification per the instructions of the manufacturer. 


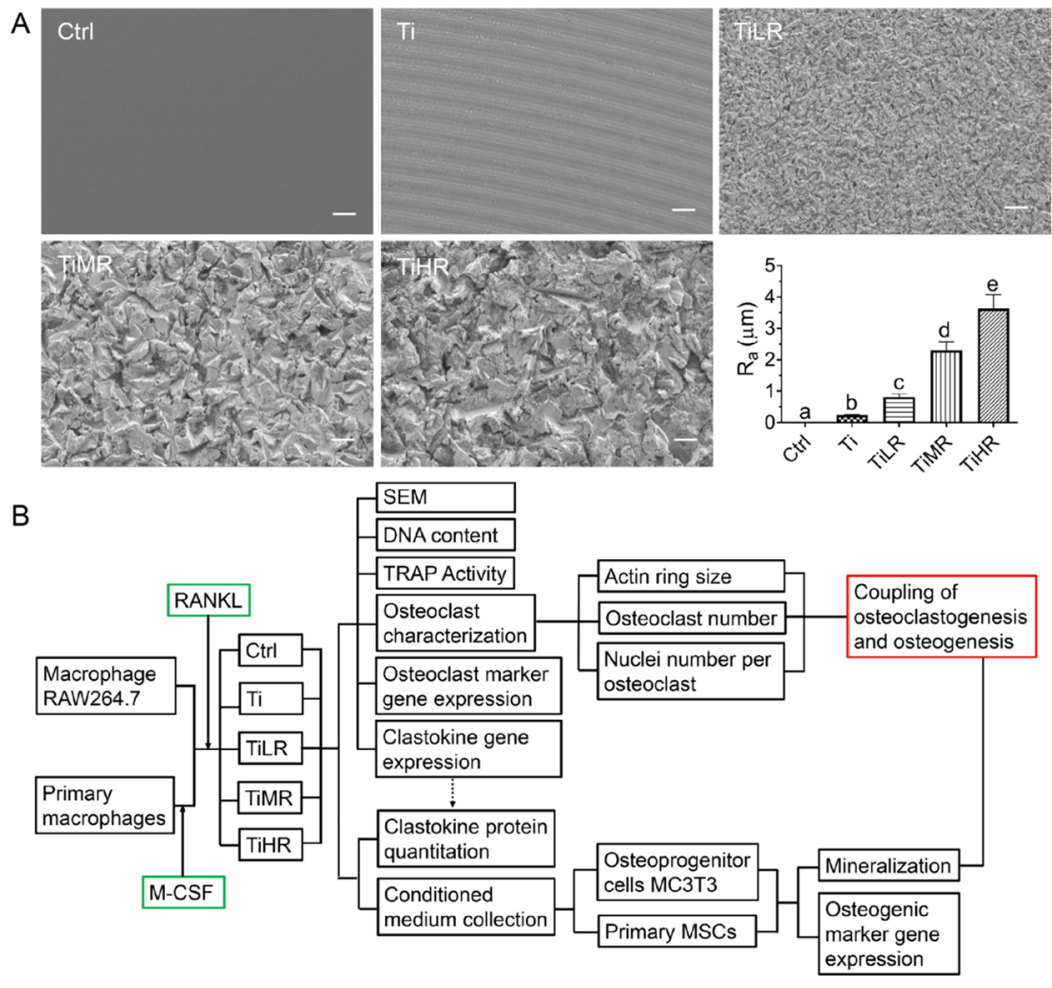

Figure 1. Surface characterization of titanium disks with different surface roughness and the flow chart for further osteoclast and osteoblast study. Machined titanium disks were sand-blasted to create different roughness, and smooth glass slides were used as control. (A) Morphology of different rough surfaces was observed by SEM. The roughness of glass and different rough titanium surfaces was determined with a UST. Original magnification for SEM $\times 500$; scale bar is $10 \mu \mathrm{m}$ in all panels. A significant difference was indicated by a, b, $c$, d, and e. Groups with different letters mean significant difference, and groups sharing the same letter are not significantly different. (B) Flow chart for osteoclast study on these different rough surfaces and further study on the interaction between osteoclasts and osteoblasts.

2.5.4. TRAP Activity Assay. Cell layers were washed twice with $\mathrm{PBS}$, rinsed with $1 \mathrm{~mL}$ of Milli-Q, and lysed by two freeze-thaw cycles. The TRAP activity was tested using the acid phosphatase activity assay kit per the manufacturer's instructions. Briefly, $50 \mu \mathrm{L}$ of the sample was mixed with $50 \mu \mathrm{L}$ of the substrate dissolved in citrate buffer and incubated at $37^{\circ} \mathrm{C}$ for $30 \mathrm{~min}$. The reactions were stopped by adding $200 \mu \mathrm{L}$ of stop solution $(0.5 \mathrm{~N} \mathrm{NaOH})$. A blank control (citrate buffer) and standard solutions were made in parallel. The absorption was measured at $405 \mathrm{~nm}$ with a multimode spectrophotometer (Biotek, Winooski, USA). The value of TRAP activity was then normalized to DNA content.

2.5.5. Gene Expression of Osteoclast Markers. RNA was isolated from osteoclasts on different surfaces using the RNA Isolation Kit per the manufacturer's protocol. Reverse transcription was performed using TaqMan Reverse Transcription kit. qPCR expression analysis was performed using a Fast SYBR Green Master Mix Kit and the PRISM 7500 sequence amplification system (Applied Biosystems, USA). TRAP, cathepsin K (CTSK), receptor activator of nuclear factor kappa-B (RANK), and matrix metallopeptidase 9 (MMP-9) were tested with GAPDH as the housekeeping gene. The primer sets used are shown in Table S1. The level of gene expression was calculated via the $2^{-\Delta \Delta C_{t}}$ method. Three independent samples were used for each gene of interest.

2.5.6. ELF97 Staining. Samples were washed twice with PBS and fixed with 4\% PFA. After fixation, the samples were washed twice with PBS. The ELF97 dye was diluted 50 times using the above acid phosphatase solution, added to each well, and incubated for $15 \mathrm{~min}$ at $37^{\circ} \mathrm{C}$ in the dark. The samples were then washed twice with PBS, and images were taken using a microscope (Zeiss AxioCam MRc5; Carl Zeiss Microimaging, Germany).

2.5.7. F-Actin and Nuclei Staining. For F-actin and nuclei staining, cells were rinsed twice with PBS and then fixed with 4\% PFA in PBS for $10 \mathrm{~min}$. After washing twice, the samples were incubated with
Alexa Fluor 568 labeled phalloidin (1:50 dilution) in PBS for 15 min. After washing with PBS, the samples were incubated with DAPI (1:500 dilution) for $5 \mathrm{~min}$ and mounted with a mounting medium. Cells were imaged with a Zeiss microscope. The osteoclast number (more than three nuclei), F-actin ring circumference, nuclei per osteoclast, and osteoclast area were quantified using Image J. The cell area was categorized into one of the following classes: $\leq 25 \times 10^{3} \mu \mathrm{m}^{2}$ per cell, $25-50 \times 10^{3} \mu \mathrm{m}^{2}$ per cell, $50-100 \times 10^{3} \mu \mathrm{m}^{2}$ per cell, $100-$ $200 \times 10^{3} \mu \mathrm{m}^{2}$ per cell, $200-400 \times 10^{3} \mu \mathrm{m}^{2}$ per cell, and $\geq 400 \times 10^{3}$ $\mu \mathrm{m}^{2}$ per surface. The nuclei number of each osteoclast was counted for four disks. If the number of osteoclasts was more than 100 per disk, the measurements were performed at random 100 osteoclasts on each specimen.

2.6. Effect of Osteoclast Medium on Osteogenic Differentiation of Osteoblastic Cells. 2.6.1. Mouse Osteoprogenitor and Rat Primary MSC Culture. The mouse osteoblastic precursor cell line MC3T3 was purchased from Sigma-Aldrich. Rat bone marrowderived MSCs were isolated from 6 week-old male Fischer rats. All animal procedures were approved by the Radboud University Nijmegen Animal Ethics Committee. Both MC3T3 cells and MSCs were cultured in the growth medium ( $\alpha$-MEM with $10 \% \mathrm{FBS}, 100 \mathrm{U} /$ $\mathrm{mL}$ penicillin, and $100 \mu \mathrm{g} / \mathrm{mL}$ streptomycin) at $37^{\circ} \mathrm{C}$ in humidified $5 \% \mathrm{CO}_{2}$.

For osteogenic differentiation assays, $2 \times 10^{4}$ cells were seeded in 48-well plates. After $24 \mathrm{~h}$, the medium was changed with a 1:1 conditioned medium from RAW264.7-derived osteoclast cultures (for MC3T3 cells) or primary mouse osteoclast cultures (for primary MSCs) on Ti disks with different roughness and osteogenic medium (OM; growth medium supplemented with $10 \mathrm{nM}$ dexamethasone, $100 \mu \mathrm{M}$ ascorbic acid, and $10 \mathrm{mM} \beta$-glycerophosphate). The medium was changed twice per week. These cultures were used to obtain samples for alizarin red staining and mineralization quantification and for gene expression analysis. 
2.6.2. Alizarin Red Staining and Quantification. After 14 days, cell layers were washed twice with PBS and then fixed with 4\% PFA for $10 \mathrm{~min}$ and then washed twice with PBS. Cells were then stained with $1 \mathrm{~mL} /$ well alizarin red solution for $15 \mathrm{~min}$ at room temperature using an osteogenesis quantification kit. Stained samples were photographed with a light microscope. The quantification of alizarin red of cell layer was conducted based on the protocol. Briefly, cell layers were dissolved in $10 \%$ acetic acid, vortexed, heated at $85{ }^{\circ} \mathrm{C}$, and then centrifuged. The supernatant was neutralized, and then colorimetric changes were measured at the absorbance of $405 \mathrm{~nm}$ using a multimode spectrophotometer (Biotek, Winooski, USA). To reduce the internal errors, alizarin red values from each sample were normalized to cells cultured in 1:1 of OM and growth medium.

2.6.3. Gene Expression of Osteogenic Markers. Osteoprogenitor cells were cultured in the conditioned medium of osteoclasts for 7 days, and RNA was isolated and transcribed as aforementioned. The gene expression of RUNX2, collagen I, ALP, and osteocalcin (OCN) was analyzed by qPCR using the primers listed in Table S1. GAPDH was used as the housekeeping gene, and the level of gene expression was calculated via the $2^{-\Delta \Delta C_{t}}$ method. Four independent samples were used for each gene of interest.

2.7. Quantification of Clastokine Gene Expression and Protein Secretion of Osteoclasts on Different Roughness. 2.7.1. Gene Expression of Clastokines. mRNA of osteoclasts on different surfaces was isolated and transcribed into cDNA as described above. qPCR expression analysis was similarly performed. SPHK1, Wnt10b, BMP-6, CTHRC1, Scl, and Sema4D were tested with $G A P D H$ as the housekeeping gene. The primer sets used are shown in Table S2. The level of gene expression was calculated via the $2^{-\Delta \Delta C_{t}}$ method. Three independent samples were used for each gene of interest.

2.7.2. Quantification of Clastokine Concentration in the Conditioned Medium. The conditioned medium of osteoclasts cultured on different surfaces were used to quantify the BMP- 6 and CTHRC1 concentration based on the protocols from ELISA kits (MyBiosource, USA).

2.8. Regression Analysis of Osteoclast Subtype and Its Anabolic Activity. Size of actin ring, number of osteoclasts per square centimeter, number of nuclei per osteoclast, and TRAP activity were averaged for each surface roughness group. These values were then correlated with the alizarin red value of osteoblastic cells cultured in each type of osteoclasts on different surfaces. Scatter diagrams were made with Excel, regression analyses were conducted, and the most accurate trend lines were displayed based on the $R$ value.

2.9. Statistical Analysis. All experiments were repeated three times, and figures show the representative data of a single representative experiment. All results are expressed as the mean \pm standard deviation from multiple samples per experimental group (see figure captions for exact sample numbers), and $P<0.05$ was considered statistically significant. One-way analysis of variance was used for each experiment to compare the means among the groups. Where applicable, a Tukey's honestly significant difference test was used as a post hoc test.

\section{RESULTS}

3.1. Preparation and Characterization of Titanium Disks with Different Surface Roughness. Four types of titanium disks were prepared with significantly different surface roughness (Figure 1A). SEM assessment of the surface morphology showed that Ti surfaces were apparently smooth. TiLR displayed a low roughness and uniform topography compared to TiMR and TiHR, which showed crack structures and blasting scars with a higher roughness (Figures 1A \& S1). The $R_{\mathrm{a}}$ values were $0.24 \mu \mathrm{m}$ for machined (Ti), $0.81 \mu \mathrm{m}$ for low roughness (TiLR), $2.30 \mu \mathrm{m}$ for medium roughness (TiMR), and $3.63 \mu \mathrm{m}$ for high roughness (TiHR). Glass slides with a smooth surface $\left(R_{\mathrm{a}}=0.02 \mu \mathrm{m}\right)$ served as controls (Ctrl). After surface characterization, the macro- phages were seeded on these surfaces and induced into osteoclasts whose features were further analyzed (Figure 1B).

3.2. Osteoclast Morphology on Different Surface Roughness. The morphology of macrophages after osteoclastogenic induction on different surfaces was observed with SEM (Figure S1; RAW264.7 macrophages were representatively displayed). Both large giant cells and smaller, undifferentiated macrophages were visible on all surfaces. The osteoclast morphology was greatly affected by the surface roughness. Osteoclasts present on the smooth surfaces of Ctrl and Ti showed a more spread shape and more clear cell fusion than cells on rougher surfaces (TiLR, TiMR, and TiHR), on which cells were smaller and separately distributed.

3.3. Osteoclast Differentiation with Different Surface Roughness. To determine the osteoclastic nature of the formed multinucleated cells, TRAP staining was performed. Different sizes and numbers of TRAP-positive cells originating from RAW264.7 macrophages were observed on different surfaces (Figure 2A). TRAP-positive cells were bigger and present in lower numbers on smoother disks ( $\mathrm{Ctrl}$ and $\mathrm{Ti}$ ) than on rougher disks. A difference between TiLR, TiMR, and TiHR was not obvious. To assess the cell numbers on these different surfaces, DNA content was measured after 4 days of osteoclastogenic induction. DNA content from RAW264.7 cells gradually increased with surface roughness. A significantly higher cell number was observed for rougher surfaces (TiMR and $\mathrm{TiHR})$ compared to smoother titanium surfaces $(\mathrm{Ctrl}, \mathrm{Ti}$, and TiLR; Figure 2B). Quantitative assessment of osteoclastic TRAP activity on different surfaces (Figure 2C) showed a tendency of smooth surfaces to have higher TRAP activity than rougher surfaces. Specifically, the highest TRAP activity was found on $\mathrm{Ti}$, followed by $\mathrm{Ctrl}$ and TiLR, which were significantly higher than TiMR and TiHR. The negative control without RANKL showed negligible TRAP activity (data not shown). To further confirm osteoclastic differentiation, a series of specific markers were analyzed by qPCR (Figure $2 \mathrm{D}-\mathrm{G}$ ). Osteoclastogenic markers were generally expressed at significantly higher levels on Ctrl and $\mathrm{Ti}$ than on rougher surfaces. Among roughened surfaces, TiLR had significantly higher RANK and MMP-9 gene expression than TiMR and TiHR.

For primary mouse osteoclasts, TRAP biochemical staining and TRAP activity displayed similar responses to the different surfaces (Figure 3). More specifically, with increasing surface roughness, primary mouse osteoclasts decreased in size on TiLR, TiMR, and TiHR compared to Ctrl and Ti. The number of TRAP-positive cells was generally higher on rougher surfaces (Figure 3A). The cell number evaluated with DNA content was also significantly higher on rougher surfaces, and TRAP activity declined with increasing roughness (Figure 3B,C). However, the TRAP activity was highest on Ctrl for primary mouse osteoclasts (in contrast to Ti for RAW264.7derived osteoclasts).

3.4. Osteoclasts Number, Size, and Cytoskeletal Organization on Different Surface Roughness. When osteoclast precursors differentiate into mature osteoclasts, they form clusters of dynamic, F-actin-rich adhesion structures enriched in integrin receptors called podosome that selforganize into actin rings at the cytomembrane periphery. ${ }^{3,34}$ We investigated the effects of surface roughness on actin ring formation by the analysis of F-actin (phalloidin stain), nuclei (DAPI stain), and endogenous phosphatase activity (ELF97 stain) organization. On all surfaces, osteoclasts from 
A

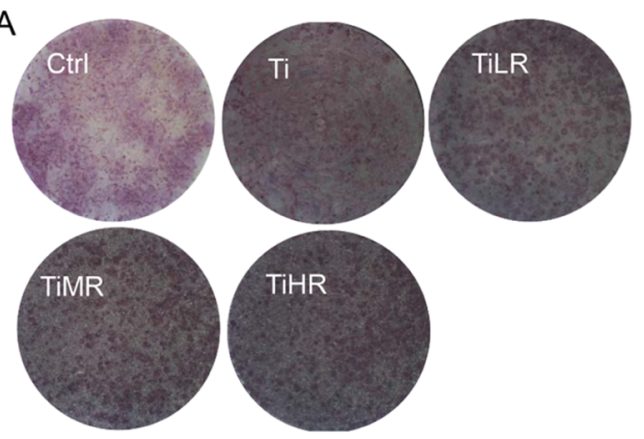

B
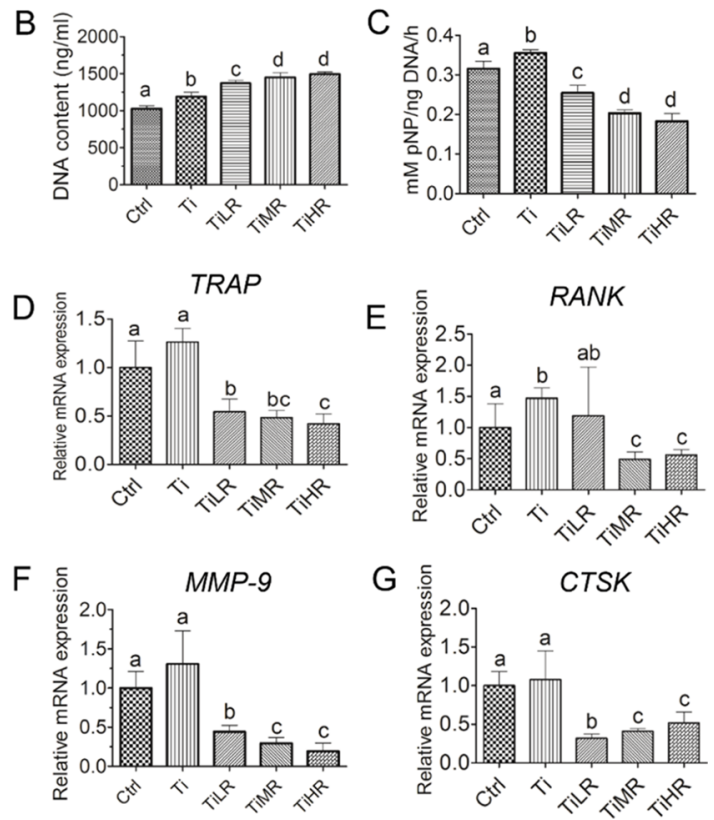

Figure 2. Osteoclastogenic differentiation of RAW264.7-derived osteoclasts on different rough surfaces. RAW264.7-derived macrophages were cultured on glass control and different rough titanium with RANKL for 4 days. (A) Osteoclasts were then stained with TRAP biochemical staining $(n=4)$. (B) Cell number on these surfaces was quantified by DNA content $(n=4)$, and their osteoclastogenic differentiation was determined by (C) TRAP activity $(n=4)$ and gene expression of osteoclast makers including (D) TRAP $(n=3)$, (E) RANK $(n=3)$, (F) MMP-9 $(n=3)$, and (G) CTSK $(n=$ 3). A significant difference was indicated by a, b, c, and d. Groups with different letters mean significant difference, and groups sharing the same letter are not significantly different.

RAW264.7 exhibited multiple nuclei, a typical F-actin ring, and endogenous phosphatase positivity (Figure 4A). Actin rings were typically big and heterogeneous on smoother surfaces. In contrast, osteoclasts cultured on rougher surfaces displayed small but homogeneous F-actin ring organization and cluster structure. To be specific, osteoclasts on Ctrl (average 1100 $\mu \mathrm{m})$ and $\mathrm{Ti}$ (average $906 \mu \mathrm{m}$ ) exhibited significantly larger actin rings in circumference than osteoclasts on TiLR (average $566 \mu \mathrm{m}$ ) and TiMR (average $491 \mu \mathrm{m}$ ) surfaces, with TiHR having the lowest size (average $358 \mu \mathrm{m}$; Figure 4B). The number of osteoclasts was quantitatively determined on the different surfaces with the aid of F-actin ring and endogenous phosphatase staining. A significantly larger number of osteoclasts were found on rougher surfaces, especially on TiHR $\left(37 \pm 3.2\right.$ osteoclasts $\left./ \mathrm{cm}^{2}\right)$ and TiMR surfaces $(26 \pm$ 2.3 osteoclasts $\left./ \mathrm{cm}^{2}\right)$, compared to the $\mathrm{Ti}(3.5 \pm 0.5$ osteoclasts $\left./ \mathrm{cm}^{2}\right)$ and Ctrl $\left(2.7 \pm 0.8\right.$ osteoclasts $\left./ \mathrm{cm}^{2}\right)$

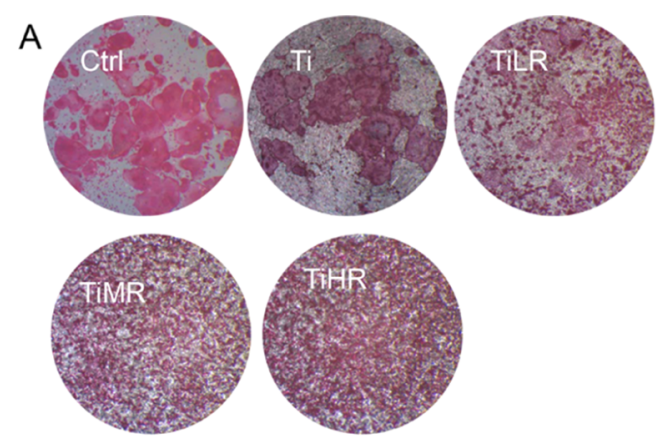

B

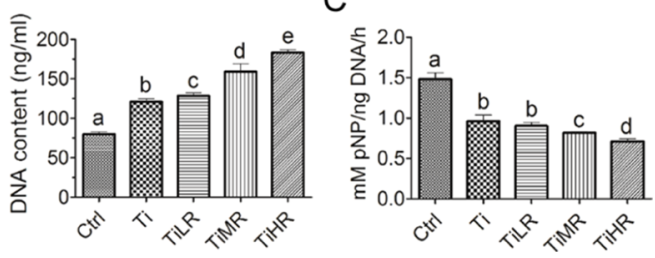

Figure 3. Osteoclastogenic differentiation of primary osteoclasts on different rough surfaces. Primary mouse macrophages were cultured on glass control and different rough titanium with M-CSF and RANKL for 4 days. (A) Osteoclasts were then stained with TRAP biochemical staining $(n=4)$. (B) Cell number on these surfaces was quantified by the DNA content $(n=4)$, and (C) their osteoclastogenic differentiation was determined by TRAP activity ( $n$ $=4)$. A significant difference was indicated by a, b, c d, and e. Groups with different letters mean significant difference, and groups sharing the same letter are not significantly different.

smoother surfaces (Figure 4C). The average nuclei number in each osteoclast, however, was significantly higher on smoother surfaces, especially on $\mathrm{Ti}$ (average 87 nuclei/ osteoclast). This was approximately 3 times more than TiLR, 4 times more than TiMR, and 8 times more than TiHR (Figure 4D). The osteoclast size, evaluated by cell area, was also analyzed based on F-actin ring and endogenous phosphatase staining. Both large and small osteoclasts were observed and measured on all surfaces, where the largest osteoclast areas were found on smoother surfaces ( $\mathrm{Ctrl}$ and $\mathrm{Ti}$; Figure 4E). More specifically, on smoother surfaces (Ctrl and $\mathrm{Ti})$, large osteoclasts with an area $>50 \times 10^{3} \mu \mathrm{m}^{2}$ were predominant, constituting more than $71 \%$ of all osteoclasts, whereas small osteoclasts with a typical area of less than $25 \times$ $10^{3} \mu \mathrm{m}^{2}$ constituted only $15 \%$ of the population. On rougher surfaces (TiLR, TiMR, and TiHR), an opposite trend was observed: osteoclasts with an area $<25 \times 10^{3} \mu \mathrm{m}^{2}$ were the predominant phenotype, constituting more than $51 \%$ of total osteoclast population, whereas large osteoclasts with an area $>50 \times 10^{3} \mu \mathrm{m}^{2}$ constituted less than $17 \%$.

Compared to RAW264.7-derived osteoclasts, primary mouse osteoclasts also generally exhibited bigger F-actin ringlike structures on smoother surfaces than rougher surfaces, except for those on Ctrl, and these cells did not display phalloidinlabeled F-acting ring formation in three independent experiments (Figure 5A). When F-actin rings were measured on other surfaces for quantitative comparison, F-actin ring sizes of primary mouse osteoclasts on $\mathrm{Ti}$ (average $1746 \mu \mathrm{m}$ ) similarly displayed a significantly larger size in circumference than those on TiLR (average $493 \mu \mathrm{m}$ ), TiMR (average $207 \mu \mathrm{m}$ ), and TiHR (average $196 \mu \mathrm{m}$; Figure 5B). The number of osteoclasts was also highest on TiHR (111 \pm 12 osteoclasts/ $\left.\mathrm{mm}^{2}\right)$, followed by TiMR $\left(65 \pm 4.1\right.$ osteoclasts $\left./ \mathrm{mm}^{2}\right)$ and 

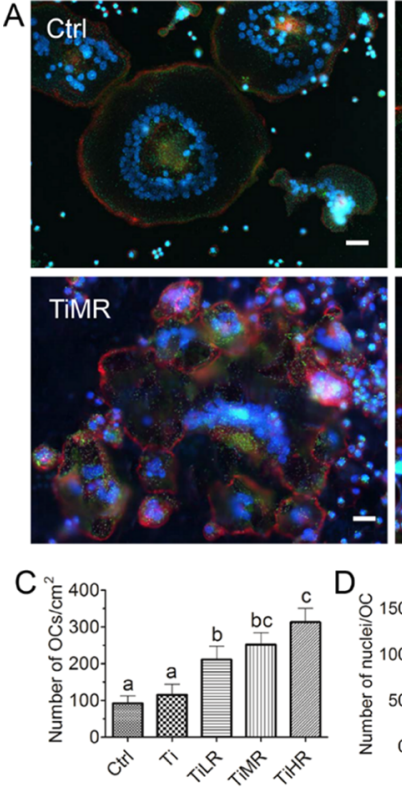
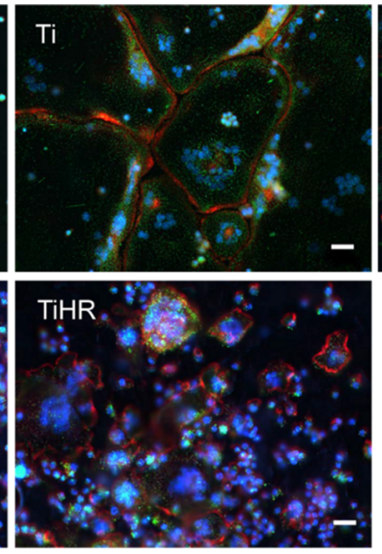

E
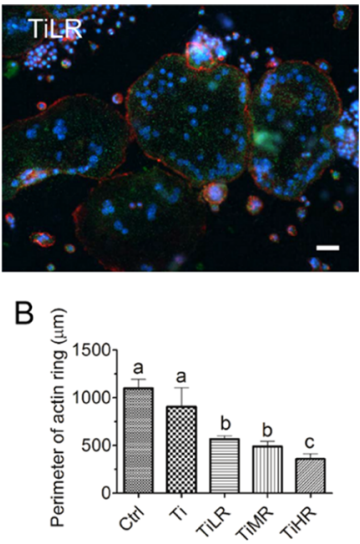
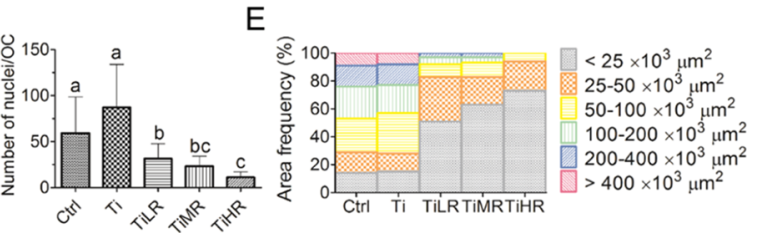

Figure 4. Number, size, and cytoskeletal organization of RAW264.7-derived osteoclasts on different rough surfaces. RAW264.7-derived macrophages were cultured on glass control and different rough titanium with RANKL for 4 days. (A) Osteoclasts were then stained with DAPI (blue), Alexa Fluor 568 Phalloidin (red), and ELF 97 (green). (B) Perimeter of actin rings, (C) osteoclasts density, (D) nuclei number per osteoclast, and (E) osteoclast area on these different surfaces were determined to quantify the osteoclastogenic differentiation. Scale bar $=50 \mu \mathrm{m}$. A significant difference was indicated by a, b, c, and d. Groups with different letters mean significant difference and groups sharing the same letter are not significantly different.

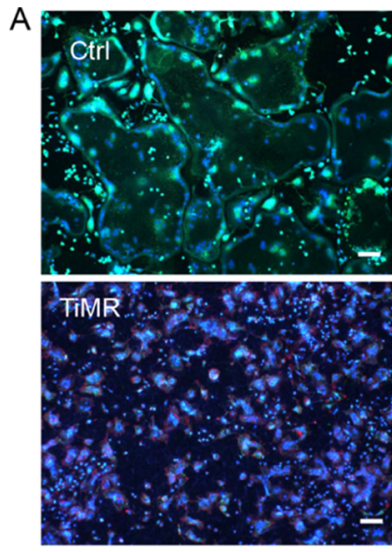

C

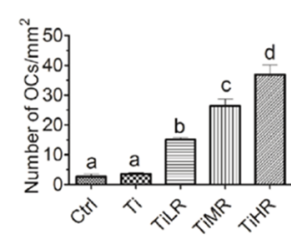

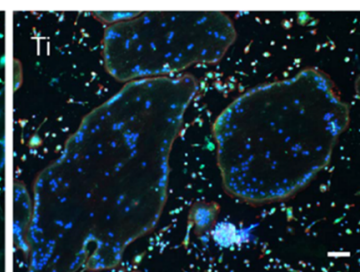

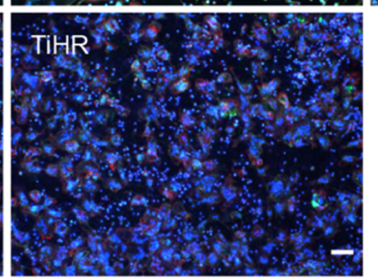

E

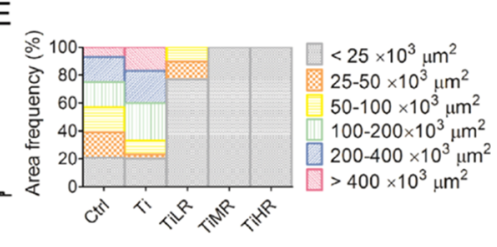

Figure 5. Number, size, and cytoskeletal organization of primary osteoclasts on different rough surfaces. Primary mouse macrophages were cultured on glass control and different rough titanium with M-CSF and RANKL for 4 days. (A) Osteoclasts were then stained with DAPI (blue), Alexa Fluor 568 Phalloidin (red), and ELF 97 (green). (B) Perimeter of actin rings, (C) osteoclasts density, (D) nuclei number per osteoclast, and (E) osteoclast area on these different surfaces were determined to quantify the osteoclastogenic differentiation. Scale bar $=100 \mu \mathrm{m}$. A significant difference was indicated by $a, b, c$, and $d$. Groups with different letters mean significant difference, and groups sharing the same letter are not significantly different.

$\operatorname{TiLR}\left(33 \pm 4.3\right.$ osteoclasts $\left./ \mathrm{mm}^{2}\right)$, all of which were significantly higher than on $\mathrm{Ti}$ and $\mathrm{Ctrl}$ (around two osteoclasts $/ \mathrm{mm}^{2}$; Figure $5 \mathrm{C}$ ). Also, primary mouse osteoclasts on $\mathrm{Ti}$ (average 198 nuclei/osteoclast) and Ctrl (average 32 nuclei/osteoclast) had significantly more nuclei per osteoclast than those on rougher surfaces (all fewer than 19 nuclei/ osteoclast; Figure 5D). Regarding cell area, primary mouse osteoclasts displayed less diversity compared to RAW264.7derived osteoclasts on all surfaces. Osteoclast areas $<25 \times 10^{3}$ $\mu \mathrm{m}^{2}$ predominated for primary mouse osteoclasts on TiLR, TiMR, and TiHR. However, primary mouse osteoclasts on Ctrl and $\mathrm{Ti}$ showed larger area, with an area $<25 \times 10^{3} \mu \mathrm{m}^{2}$ taking up less than $17 \%$ of the osteoclasts and an area $>50 \times 10^{3} \mu \mathrm{m}^{2}$ approximately constituting $60 \%$ of the osteoclasts (Figure $5 \mathrm{E}$ ). 

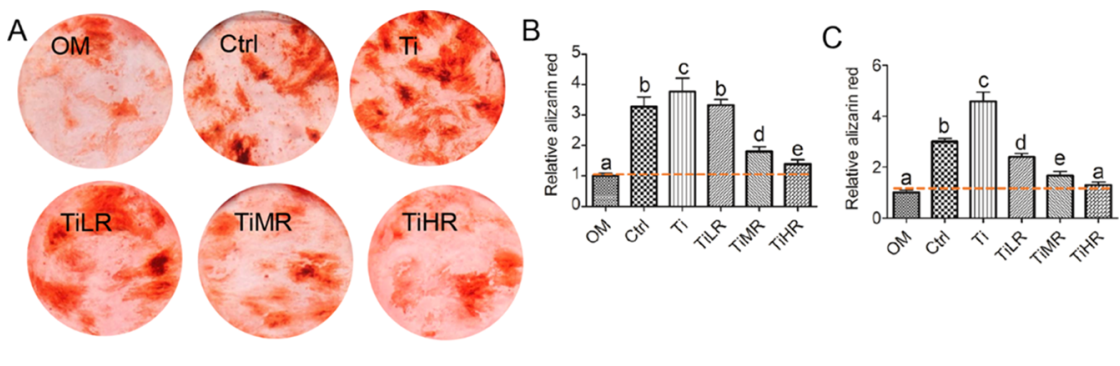

D
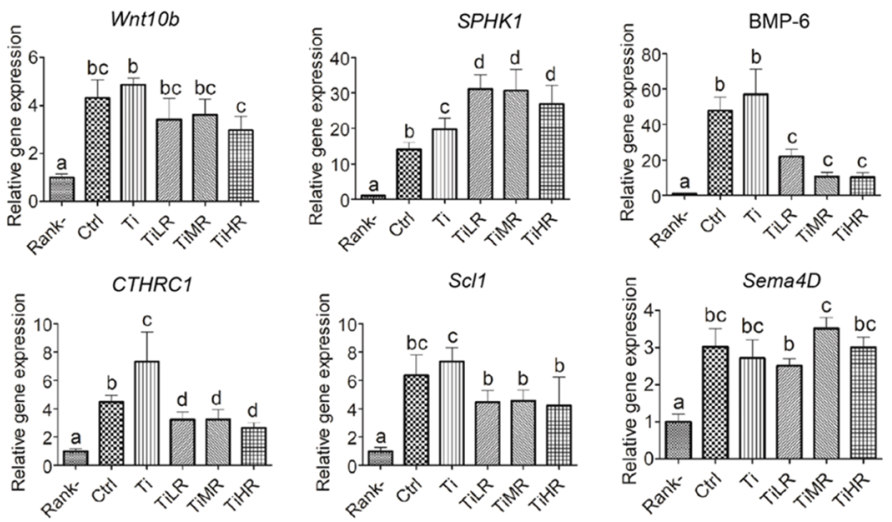

E
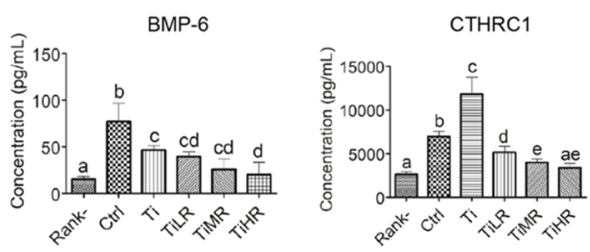

Figure 6. Anabolic effects and potential clastokines of conditioned medium of osteoclasts cultured on different rough surfaces. Mouse osteoprogenitor cells (MC3T3) and primary rat MSCs were cultured in the conditioned medium of osteoclasts cultured on glass control and different titanium rough surfaces and OM $(50 \% \mathrm{v} / \mathrm{v})$. (A) Mineralization nodules were stained with alizarin red and quantified with alizarin red (B) of mouse osteoprogenitor cells in a conditioned medium from RAW264.7-derived osteoclasts and (C) of primary rat MSCs in a conditioned medium from primary mouse osteoclasts. (D) Gene expression of six potential clastokines for osteoclasts on different surfaces and (E) protein concentration of two potential clastokines in a conditioned medium of RAW264.7-derived osteoclasts were quantified. A significant difference was indicated by $a, b, c$ d, and e. Groups with different letters mean significant difference, and groups sharing the same letter are not significantly different.

3.5. Effect of Osteoclast Medium on Osteogenic Differentiation of Osteoblastic Cells. Given that osteoclast differentiation and cytoskeletal organization are affected by surface roughness, we hypothesized that these roughnessinduced different subtypes of osteoclasts may further differentially regulate the behavior of osteoblastic cells by secreting specific coupling cytokines/clastokines. To this end, both mice osteoprogenitor cells and primary rat MSCs were cultured in the medium collected from the osteoclasts cultured on the surface with different roughness mixed with $\mathrm{OM}(50 \% \mathrm{v} / \mathrm{v}$; Figure 6). Osteoblastic cells subjected to conditioned medium harvested from osteoclasts displayed significantly higher alizarin red positive bone nodule formation than osteoblastic cells in regularly used OM (Figure 6A). More specifically, when MC3T3 osteoblastic cells were cultured with RAW264.7derived osteoclast-conditioned medium, the medium from osteoclasts cultured on Ctrl, Ti, and TiLR showed 3 times more alizarin red than osteoblast control cultures in OM. Further, the osteoclast-conditioned medium from TiMR and TiHR showed twice more alizarin red than osteoblast control cultures, and this effect was more obvious on TiMR (Figure 6B). This anabolic effect on osteoblastic cells was also observed for conditioned medium from primary osteoclasts on primary rat MSCs (Figure 6C). The conditioned medium from osteoclasts cultured on all surfaces except TiHR exhibited significantly higher alizarin red nodule formation than osteoblast control cultures in OM. Particularly, the conditioned medium from $\mathrm{Ti}$ induced approximately 5 times higher mineralization than osteoblast control cultures and 2.5 times higher than TiLR and TiMR. In agreement with our observations on mineralization, MC3T3 osteoblastic cells cultured in conditioned medium collected from RAW264.7derived osteoclasts on Ctrl and $\mathrm{Ti}$ had significantly higher osteogenic marker gene expression than those from TiMR and TiHR, including ALP, collagen I, and OCN (Figure S2).

3.6. Identification of Potential Clastokines in a Conditioned Medium of Osteoclasts on Different Surfaces. To further elucidate the potential cytokines involved in the anabolic effects of osteoclasts on osteoblastic cells, we quantified the gene expression of six reported clastokines of osteoclasts on different rough surfaces (Figure 6D). Compared to macrophages (RANKL-), all these clastokines were upregulated for osteoclasts on different surfaces, in particular, BMP-6 (10-57 times) and SPHK1 (14-31 times). Regarding the difference between osteoclasts on different rough surfaces, BMP-6 and CTHRCl were 
significantly upregulated, whereas SPHK1 was significantly downregulated for osteoclasts on Ctrl and Ti compared to that on TiLR, TiMR, and TiHR. Given the enhanced osteogenic differentiation of MSCs in the conditioned medium of osteoclasts on $\mathrm{Ctrl}$ and $\mathrm{Ti}$, we further quantified the protein levels of BMP-6 and CTHRC1 in the conditioned medium (Figure 6E). Correspondingly, we found a significantly higher concentration of CTHRC1 in the conditioned medium of osteoclasts formed on $\mathrm{Ti}$ and $\mathrm{Ctrl}$ compared to other conditioned media. Regarding BMP-6, we observed a significantly higher concentration in the conditioned medium from osteoclasts formed on Ctrl compared to Ti, TiLR, TiMR, and TiHR.

3.7. Regression Analysis between Osteoclast Characteristics and Their Anabolic Effects on Osteoblastic Cells. In order to further reveal the dependent relationship of osteoclast anabolic effects and osteoclast subtypes induced by roughness, we correlated the main differentiation parameters of osteoclast subtypes (i.e., average osteoclast number, average nuclei number per osteoclast, average osteoclast perimeter, and TRAP activity) with the mineralization content of osteoblastic cells cultured in the conditioned medium from these different osteoclast subtypes. For both RAW264.7-derived osteoclasts and primary mouse osteoclasts, osteoclast anabolic effects were found to correlate with osteoclast nuclei number (correlation coefficient $R \geq 0.95$; Figure 7A,B). Osteoclast nuclearity was found to logarithmically correlate with the anabolic effects of the osteoclast-conditioned medium on osteoblastic cultures.

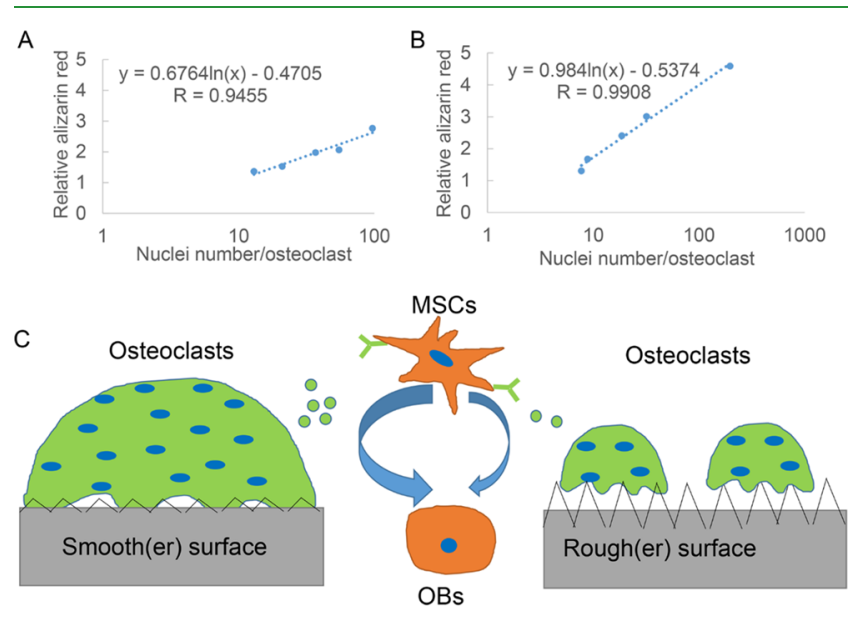

Figure 7. Regression analysis between osteoclast phenotype and its anabolic effects and the schematic of the hypothesis of interactions between osteoblastic cells, osteoclasts, and surface roughness. The average nuclei number of each osteoclast on different surfaces was correlated with its anabolic effects on osteoblastic cells. Both (A) average nuclei number of osteoclasts from RAW264.7 and their anabolic effects on mouse osteoprogenitor cells and (B) average nuclei number of primary mouse osteoclasts and their anabolic effects on primary rat MSCs displayed a logarithmic correlation, respectively. (C) On the basis of our findings of different osteoclast phenotypes and their anabolic effects, the hypothesis of the effect of roughness on osteoclastogenic differentiation and further osteogenic differentiation was proposed. On smoother surfaces, osteoclasts exhibit larger size and more nuclei in each osteoclast and secrete more anabolic clastokines to promote osteogenic differentiation of MSCs. In contrast, on rougher surfaces, osteoclasts exhibit smaller size and fewer nuclei in each osteoclast and secrete less anabolic clastokines to promote osteogenic differentiation. Green dots indicate potential clastokines (such as CTHRC1) secreted from osteoclasts.
The number of osteoclasts per square centimeter also showed correlation with osteoblastic cell mineralization (correlation coefficient of $R \geq 0.74$; Figure S3A,B). Nonetheless, the correlation of primary mouse osteoclast actin ring size and the TRAP activity with its osteoclast anabolic effects was not obvious (Figure S3C-F).

\section{DISCUSSION}

Surface properties play an important role in regulating cell and tissue responses to bone and dental implants and further determine the bone formation and osseointegration. ${ }^{10,35}$ The cross-talk between surface roughness and bone resorption cell osteoclasts and subsequent bone-forming osteoblastic cells is of great importance but remains unclear. In this study, we demonstrated that osteoclasts are sensitive to the specific surface roughness and exhibit various phenotypes, characterized by different morphology and differentiation capacity. The conditioned medium from these different osteoclastic phenotypes further promoted the osteogenic differentiation of osteoblastic cells to different degrees. The tight correlation of osteoclast nuclei number and secreted clastokines with its anabolic effect of conditioned medium from osteoclasts with this phenotype was identified. This information highlights the importance of surface properties toward osteoclast phenotype and function, which differentially orchestrates osteogenic differentiation of osteoblastic cells via secreted clastokines. Additionally, the formation of osteoclasts with specific phenotypes on surfaces with different physical properties may provide a potential guide for developing biomedical devices with an optimal surface to stimulate osseointegration.

Both RAW264.7 and primary bone marrow derived macrophages formed osteoclasts upon stimulation with RANKL, evidenced by the presence of a cell population displaying TRAP activity, the presence of multinucleated cells with well-defined actin rings, and the expression of the osteoclast-related genes TRAP, RANK, CATK, and MMP-9. Another multinucleated cell type, the so-called foreign-body multinucleated giant cells (FBGCs), are frequently formed at implant surfaces and related to foreign-body reactions. Similar to osteoclasts, FBGCs arise from the fusion of monocytes/ macrophages and hence share several characteristics with osteoclasts, such as multinuclearity, TRAP expression, and actin rings. ${ }^{36}$ An important distinction between these two cell types on nonmineral surfaces is that only osteoclasts express the matrix-degrading enzyme CTSK. ${ }^{36}$ The high gene expression of CTSK here indicates the formation of osteoclasts rather than FBGCs on all surfaces in this work.

After cell seeding, significantly higher cell number was observed for rougher surfaces than for smoother surfaces, both for RAW264.7-derived osteoclasts and primary mouse macrophage-derived osteoclasts. This finding is consistent with previous reports, in which rougher surfaces had more cells than smoother surfaces. ${ }^{37,38}$ Regarding osteoclast differentiation on different surfaces, smoother surfaces are generally more robustly induced osteoclast differentiation than rougher surfaces, evidenced by significantly higher TRAP activity and higher gene expression of osteoclastogenic markers. This difference was supposed to be bigger for different osteoclasts, given that undifferentiated macrophages were present with osteoclasts on different surfaces. This trend is in line with previous studies reporting that the increase in surface roughness $(1-2 \mu \mathrm{m})$ decreased osteoclast-associated features, $^{39,40}$ including TRAP activity and resorption capacity. 
However, it has also been observed that osteoclasts display an increased differentiation on increasing surfaces $(0.1-0.5 \mu \mathrm{m})$, measured as TRAP activity and specific gene expression. ${ }^{37}$ The different roughness range, high variety of osteoclast cellular origins, osteoclast culture protocols, and induction times may attribute to these conflicting conclusions. For example, primary osteoclasts were utilized and collected after a 21 day induction period by Costa-Rodrigues et al. ${ }^{37}$ However, as reported and proven in our study, osteoclasts appeared as early as 4 days after induction. Additionally, a difference between primary and RAW264.7-derived osteoclasts existed as primary osteoclasts could be grown for 7 days while apoptosis was apparent in RAW264.7-derived osteoclasts after 5 days of culture because of extremely high cell density. This is one of the reasons why we used two cell types to obtain osteoclasts, a cell line and primary cells, to obtain a reliable conclusion here.

Osteoclast functionality depends on its tight adhesion to the bone surface, which is mediated via an actin-rich integrin adhesion structure known as the podosome belt. Podosome belt formation and turnover are highly sensitive to the local environment. ${ }^{27}$ Generally, larger actin rings were found on smoother surfaces and small actin rings were found on rougher surfaces. One interesting finding is that primary osteoclasts on glass controls did not display this featured structure, perhaps because of a short-lived actin ring formation. ${ }^{41}$ Actin ring on glass probably early appeared and disappeared after 4 days of osteoclastogenic induction. This finding further emphasizes the importance of culture protocols and treatment time when studying osteoclasts. Except for the difference in actin ring formation, we also found different phenotypes of osteoclasts on surfaces with different roughness. Generally, larger sized, a higher number of nuclei per osteoclast but lower osteoclast numbers were found on smoother surfaces than on rougher surfaces. This difference plausibly can be attributed to the effects of roughness on the cell fusion process required for osteoclast formation. On smoother surfaces, no topographical features are present to hinder this cell fusion process. Hence, cells effectively fuse to form fewer osteoclasts with a larger size on these surfaces. In contrast, on the rough surfaces, the topographic features might hinder cell fusion process, leading to more osteoclasts with a smaller size. ${ }^{42}$ Intriguingly, our findings of different osteoclast morphology correspond with some observations of osteoclasts in vivo. Two types of morphologically different osteoclasts are observed in mice. ${ }^{43}$ One type exhibits an abundant "foamy", acidophilic staining of the cytoplasm and large oval vesicular nuclei. This type of osteoclast is abundant in young mice and at fracture sites in both young and old mice. The second osteoclast type exhibits considerably altered morphological characteristics and is smaller in size. These cells are found to be more abundant at the metaphysis of older animals. Furthermore, a significant shift from the former type to latter type occurs with increasing age. Therefore, it is tenable to conclude that different types may represent different functional states of osteoclasts. The osteoclasts with a larger size in young mice may have stronger anabolic but less resorption activity, whereas osteoclasts with smaller size may have weaker anabolic but stronger resorption activity. This idea is reinforced by the finding that osteoclasts with large surface area correspond to nonresorbing osteoclasts, whereas smaller osteoclasts correspond to actively resorbing osteoclasts. ${ }^{44-46}$ This hypothesis then promoted us to further explore the effect of roughness-induced osteoclast phenotypes on their anabolic activity.
Despite the observation that resorption and bone formation are coupled and implants induce the formation of multinuclear osteoclast-like cells around the new bone area, ${ }^{47,48}$ the relation between osteoclasts and bone regeneration using bone implants is rarely reported. The conventional consensus regarding the relation between bone resorption and bone formation is that specific factors such as TGF- $\beta$ and IGF-1 stored in the bone matrix are released and activated during bone resorption and then promote the osteogenic differentiation. ${ }^{49,50}$ This hypothesis is now being challenged by findings that nonresorbing osteoclasts still possess the ability to support bone formation, ${ }^{51-53}$ suggesting that osteoclast anabolic effects might not correlate directly with osteoclast resorption activity, but merely with the presence of osteoclasts. Several "clastokines" that regulate osteoblastic cell behavior have been identified. The data in present study corroborate the concept of nonresorbing osteoclasts on titanium and glass to have the potential to secrete certain clastokines that promote osteogenic differentiation. Among those clastokines, S1P (encoded by the SPHK1 gene), ${ }^{54,53}$ Wnt10b, ${ }^{54}$ BMP-6, ${ }^{54}$ and $\mathrm{CTHRC}^{56}$ have been well documented to promote the osteogenesis, whereas $\mathrm{Scl}^{32}$ and Sema4D ${ }^{31}$ were reported to inhibit the osteogenesis. We quantified the gene expression of these six clastokines and found BMP-6 and CTHRC1 were significantly upregulated for osteoclasts on smoother surfaces compared to that on rougher surfaces. Protein quantification further depicted a significantly higher concentration of CTHRC1 in the conditioned medium of osteoclasts on smoother surfaces than that on rougher surfaces. These data indicate that CTHRC1 plays a crucial role in the combinatorial surface roughness effects on osteoclastogenesis and osteogenesis.

We here observed that surface roughness affects osteogenic differentiation through a cross-talk between osteoclast activity and osteoblastic cell activity. Smoother surfaces promoted osteoclastogenic differentiation, and conditioned medium from osteoclast cultures thereon highly promoted the osteogenic differentiation of osteoblast progenitors. In contrast, rougher surface evoked less osteoclastic differentiation, and conditioned medium from osteoclast culture thereon promoted the osteogenic differentiation of osteoblast progenitors to a lesser extent (Figure 7C). As osteoclasts have shown to appear much earlier than osteoblastic cells around implants, ${ }^{22,23}$ it is reasonable to hypothesize that the effects of bone implant surface properties on osteoclasts and then on osseointegration overweighs the effects of sole surface roughness directly on osteoblastic cells. Another hypothesis is that roughness modulates osteogenic differentiation through two separate ways: indirect effects on osteoblastic cells through osteoclasts and direct effects on osteoblastic cells. In this perspective, both roughness and osteoclast phenotype affect osteoblastic cell migration, spreading, and differentiation, and in turn, both roughness and osteoblastic cell differentiation affect osteoclast behavior. ${ }^{57,58}$ In vivo, the optimized roughness for osteogenic differentiation should be the sum effect from these two (indirect and direct effects). In vitro, osteoblastic cell differentiation has shown to be optimal on microrough surfaces $\left(R_{\mathrm{a}}=3-4 \mu \mathrm{m}\right)$ compared to smooth surfaces, whereas osteoclast activity demonstrated to be higher on smooth than on microrough surfaces. ${ }^{39}$ Therefore, the optimal roughness for osseointegration was proposed to be $1-1.5$ $\mu \mathrm{m} .{ }^{11}$ However, this assumption should be validated through well-designed animal studies. Whatever the mechanism is, our 
information further strengthens the previously proposed idea that maintaining nonresorbing osteoclasts on metallic or plastic implant surface, ${ }^{59,60}$ as opposed to reducing osteoclast numbers, leads to increased bone formation, bone volume, and ultimately higher bone strength in vivo as sources of anabolic molecules for the osteoblasts. ${ }^{61}$

The most innovative finding in this study was the identification of the different osteoclast phenotypes and their differential anabolic effects, which we observed via osteoblastic cell cultures in conditioned medium. Various types of osteoclasts have been observed in physiological and pathological situations in vivo; ${ }^{62}$ their bone catabolic and anabolic functions, which crucially determine the bone quality and treatment effects, remains unclear and debatable. We first revealed that the osteoclast nuclei number positively correlates with these anabolic effects. This information might greatly aid the future bone implant design, which can optimize physical surface properties to induce desired osteoclast phenotypes and enhance osseointegration. In addition, given the reported heterogeneity of osteoclasts under different physiological conditions [e.g., osteopetrosis, osteoporosis, osteonecrosis, and (rheumatoid) arthritis] and their differential effects on the balance between bone resorption and bone formation, the finding that anabolic effects are associated with osteoclast heterogeneity and nuclei number would help to elucidate the function of observed osteoclast types in these diseases contribute to the design and development of specific therapeutic drugs for the treatment of these diseases. ${ }^{62}$

\section{CONCLUSIONS}

We here examined the combinatorial effects of surface roughness on osteoclast behavior and anabolic effects on osteogenic differentiation of osteoblast progenitors. Osteoclasts cultured on smoother surfaces showed larger size and actin rings, and more nuclei per osteoclast compared to osteoclasts cultured on rougher surfaces. All conditioned medium from these different osteoclast phenotypes significantly promoted the osteogenic differentiation of osteoblastic cells compared to conventional OM, and this effect was far more obvious for conditioned medium from osteoclasts cultured on smoother surfaces. This anabolic effect of conditioned medium on osteogenic differentiation was further revealed to logarithmically correlate with the number of nuclei per osteoclast and the presence of clastokine CTHRC1. These results suggest that surface roughness is an important factor in mediating osteoclast-material interactions, which might determine the osteogenic differentiation of osteoblast progenitor cells and hence the process of osseointegration.

\section{ASSOCIATED CONTENT}

\section{(5) Supporting Information}

The Supporting Information is available free of charge on the ACS Publications website at DOI: 10.1021/acsami.8b10992.

qPCR primer sequences of target genes for osteoclast differentiation and osteogenic differentiation and $\mathrm{qPCR}$ primer sequences of clastokines, morphologies of different rough surfaces and osteoclasts induced on these different surfaces, gene expression of osteogenic markers in mouse osteoprogenitor cells cultured in conditioned medium of different types of osteoclasts, and the coupling between osteoclast phenotype and its anabolic effects (PDF)

\section{AUTHOR INFORMATION}

\section{Corresponding Author}

*E-mail: Jeroen.vandenbeucken@radboudumc.nl. ORCID

Yang Zhang: 0000-0002-7864-1452

Jeroen J. J. P. van den Beucken: 0000-0003-2851-6896

\section{Notes}

The authors declare no competing financial interest.

\section{ACKNOWLEDGMENTS}

This study was supported by the Chinese Scholarship Council (no. 201306990006). The authors are very grateful to Dr. Zetao Chen from Queensland University Technology for providing the primer sequences used for osteoclast differentiation.

\section{REFERENCES}

(1) Narushima, M.; Nakai, T.; Niinomi, M. Advances in Metallic Biomaterials: Processing and Applications; Springer: Sendai, 2015.

(2) Niinomi, M.; Nakai, M.; Hieda, J. Development of New Metallic Alloys for Biomedical Applications. Acta Biomater. 2012, 8, 38883903.

(3) Osman, R.; Swain, M. A Critical Review of Dental Implant Materials with an Emphasis on Titanium Versus Zirconia. Materials 2015, 8, 932-958.

(4) Elias, C. N.; Lima, J. H. C.; Valiev, R.; Meyers, M. A. Biomedical Applications of Titanium and Its Alloys. JOM 2008, 60, 46-49.

(5) Esposito, M.; Hirsch, J.-M.; Lekholm, U.; Thomsen, P. Biological Factors Contributing to Failures of Osseointegrated Oral Implants, (I). Success Criteria and Epidemiology. Eur. J. Oral Sci. 1998, 106, $527-551$.

(6) Fugazzotto, P. A. Success and Failure Rates of Osseointegrated Implants in Function in Regenerated Bone for 72 to 133 Months. Int. J. Oral Maxillofac. Implants 2005, 20, 77-83.

(7) dos Santos, M. C. L. G.; Campos, M. I. G.; Line, S. R. P. Early Dental Implant Failure: A Review of the Literature. Braz. J. Oral Sci. 2002, 1, 103-111.

(8) Athanasou, N. A. The Pathobiology and Pathology of Aseptic Implant Failure. Bone Jt. Res. 2016, 5, 162-168.

(9) Zhou, R.; Wei, D.; Cao, J.; Feng, W.; Cheng, S.; Du, Q.; Li, B.; Wang, Y.; Jia, D.; Zhou, Y. Synergistic Effects of Surface Chemistry and Topologic Structure from Modified Microarc Oxidation Coatings on Ti Implants for Improving Osseointegration. ACS Appl. Mater. Interfaces 2015, 7, 8932-8941.

(10) Jemat, A.; Ghazali, M. J.; Razali, M.; Otsuka, Y. Surface Modifications and Their Effects on Titanium Dental Implants. BioMed Res. Int. 2015, 2015, 791725.

(11) Shalabi, M. M.; Gortemaker, A.; Van't Hof, M. A.; Jansen, J. A.; Creugers, N. H. J. Implant Surface Roughness and Bone Healing: A Systematic Review. J. Dent. Res. 2006, 85, 496-500.

(12) Wennerberg, A.; Albrektsson, T. Suggested Guidelines for the Topographic Evaluation of Implant Surfaces. Int. J. Oral Maxillofac. Implants 2000, 15, 331-344.

(13) Wennerberg, A.; Albrektsson, T.; Andersson, B. Design and Surface Characteristics of 13 Commercially Available Oral Implant Systems. Int. J. Oral Maxillofac. Implants 1993, 8, 119-137.

(14) Khang, W.; Feldman, S.; Hawley, C. E.; Gunsolley, J. A MultiCenter Study Comparing Dual Acid-Etched and Machined-Surfaced Implants in Various Bone Qualities. J. Periodontol. 2001, 72, 13841390.

(15) Zechner, W.; Trinkl, N.; Watzak, G.; Busenlechner, D.; Tepper, G.; Haas, R.; Watzek, G. Radiologic Follow-up of Peri-Implant Bone Loss around Machine-Surfaced and Rough-Surfaced Interforaminal Implants in the Mandible Functionally Loaded for 3 to 7 Years. Int. J. Oral Maxillofac. Implants 2004, 19, 216-221. 
(16) Trisi, P.; Lazzara, R.; Rebaudi, A.; Rao, W.; Testori, T.; Porter, S. S. Bone-Implant Contact on Machined and Dual Acid-Etched Surfaces after 2 Months of Healing in the Human Maxilla. J. Periodontol. 2003, 74, 945-956.

(17) Faia-Torres, A. B.; Guimond-Lischer, S.; Rottmar, M.; Charnley, M.; Goren, T.; Maniura-Weber, K.; Spencer, N. D.; Reis, R. L.; Textor, M.; Neves, N. M. Differential Regulation of Osteogenic Differentiation of Stem Cells on Surface Roughness Gradients. Biomaterials 2014, 35, 9023-9032.

(18) Gittens, R. A.; McLachlan, T.; Olivares-Navarrete, R.; Cai, Y.; Berner, S.; Tannenbaum, R.; Schwartz, Z.; Sandhage, K. H.; Boyan, B. D. The Effects of Combined Micron-/Submicron-Scale Surface Roughness and Nanoscale Features on Cell Proliferation and Differentiation. Biomaterials 2011, 32, 3395-3403.

(19) Lee, E. M.; Smith, K.; Gall, K.; Boyan, B. D.; Schwartz, Z. Change in Surface Roughness by Dynamic Shape-Memory Acrylate Networks Enhances Osteoblast Differentiation. Biomaterials 2016, $110,34-44$.

(20) Shen, X.; Hu, Y.; Xu, G.; Chen, W.; Xu, K.; Ran, Q.; Ma, P.; Zhang, Y.; Li, J.; Cai, K. Regulation of the Biological Functions of Osteoblasts and Bone Formation by Zn-Incorporated Coating on Microrough Titanium. ACS Appl. Mater. Interfaces 2014, 6, 1642616440.

(21) Sennerby, L.; Thomsen, P.; Ericson, L. E. Early Tissue Response to Titanium Implants Inserted in Rabbit Cortical Bone. J. Mater. Sci.: Mater. Med. 1993, 4, 240-250.

(22) Ohtsu, A.; Kusakari, H.; Maeda, T.; Takano, Y. A Histological Investigation on Tissue Responses to Titanium Implants in Cortical Bone of the Rat Femur. J. Periodontol. 1997, 68, 270-283.

(23) Orsini, E.; Salgarello, S.; Martini, D.; Bacchelli, B.; Quaranta, M.; Pisoni, L.; Bellei, E.; Joechler, M.; Ottani, V. Early Healing Events around Titanium Implant Devices with Different Surface Microtopography: A Pilot Study in an in Vivo Rabbit Model. Sci. World J. 2012, 2012, 349842.

(24) Davies, J. E.; Ajami, E.; Moineddin, R.; Mendes, V. C. The Roles of Different Scale Ranges of Surface Implant Topography on the Stability of the Bone/Implant Interface. Biomaterials 2013, 34, $3535-3546$

(25) Boyan, B. D.; Schwartz, Z.; Lohmann, C. H.; Sylvia, V. L.; Cochran, D. L.; Dean, D. D.; Puzas, J. E. Pretreatment of Bone with Osteoclasts Affects Phenotypic Expression of Osteoblast-Like Cells. J. Orthop. Res. 2003, 21, 638-647.

(26) Hefti, T.; Frischherz, M.; Spencer, N. D.; Hall, H.; Schlottig, F. A Comparison of Osteoclast Resorption Pits on Bone with Titanium and Zirconia Surfaces. Biomaterials 2010, 31, 7321-7331.

(27) Saltel, F.; Chabadel, A.; Bonnelye, E.; Jurdic, P. Actin Cytoskeletal Organisation in Osteoclasts: A Model to Decipher Transmigration and Matrix Degradation. Eur. J. Cell Biol. 2008, 87, 459-468.

(28) Blair, H.; Teitelbaum, S.; Ghiselli, R.; Gluck, S. Osteoclastic Bone Resorption by a Polarized Vacuolar Proton Pump. Science 1989, 245, 855-857.

(29) Sims, N. A.; Martin, T. J. Coupling the Activities of Bone Formation and Resorption: A Multitude of Signals within the Basic Multicellular Unit. Bonekey Rep 2014, 3, 481.

(30) Teti, A. Mechanisms of Osteoclast-Dependent Bone Formation. Bonekey Rep 2013, 2, 449.

(31) Negishi-Koga, T.; Shinohara, M.; Komatsu, N.; Bito, H.; Kodama, T.; Friedel, R. H.; Takayanagi, H. Suppression of Bone Formation by Osteoclastic Expression of Semaphorin 4d. Nat. Med. 2011, 17, 1473-1480.

(32) Kusu, N.; Laurikkala, J.; Imanishi, M.; Usui, H.; Konishi, M.; Miyake, A.; Thesleff, I.; Itoh, N. Sclerostin Is a Novel Secreted Osteoclast-Derived Bone Morphogenetic Protein Antagonist with Unique Ligand Specificity. J. Biol. Chem. 2003, 278, 24113-24117.

(33) Georgess, D.; Machuca-Gayet, I.; Blangy, A.; Jurdic, P. Podosome Organization Drives Osteoclast-Mediated Bone Resorption. Cell Adhes. Migr. 2014, 8, 192-204.
(34) Destaing, O.; Saltel, F.; Géminard, J.-C.; Jurdic, P.; Bard, F. Podosomes Display Actin Turnover and Dynamic Self-Organization in Osteoclasts Expressing Actin-Green Fluorescent Protein. Mol. Biol. Cell 2003, 14, 407-416.

(35) Li, G.; Cao, H.; Zhang, W.; Ding, X.; Yang, G.; Qiao, Y.; Liu, X.; Jiang, X. Enhanced Osseointegration of Hierarchical Micro/ Nanotopographic Titanium Fabricated by Microarc Oxidation and Electrochemical Treatment. ACS Appl. Mater. Interfaces 2016, 8, $3840-3852$.

(36) ten Harkel, B.; Schoenmaker, T.; Picavet, D. I.; Davison, N. L.; de Vries, T. J.; Everts, V. The Foreign Body Giant Cell Cannot Resorb Bone, but Dissolves Hydroxyapatite Like Osteoclasts. PLoS One 2015, 10, No. e0139564.

(37) Costa-Rodrigues, J.; Fernandes, A.; Lopes, M. A.; Fernandes, M. H. Hydroxyapatite Surface Roughness: Complex Modulation of the Osteoclastogenesis of Human Precursor Cells. Acta Biomater. 2012, 8, 1137-1145

(38) Makihira, S.; Mine, Y.; Kosaka, E.; Nikawa, H. Titanium Surface Roughness Accelerates Rankl-Dependent Differentiation in the Osteoclast Precursor Cell Line Raw264.7. Dent. Mater. J. 2007, 26, 739-745.

(39) Costa, D. O.; Prowse, P. D. H.; Chrones, T.; Sims, S. M.; Hamilton, D. W.; Rizkalla, A. S.; Dixon, S. J. The Differential Regulation of Osteoblast and Osteoclast Activity by Surface Topography of Hydroxyapatite Coatings. Biomaterials 2013, 34, $7215-7226$.

(40) Brinkmann, J.; Hefti, T.; Schlottig, F.; Spencer, N. D.; Hall, H. Response of Osteoclasts to Titanium Surfaces with Increasing Surface Roughness: An in Vitro Study. Biointerphases 2012, 7, 34.

(41) Geblinger, D.; Addadi, L.; Geiger, B. Nano-Topography Sensing by Osteoclasts. J. Cell Sci. 2010, 123, 1503-1510.

(42) Geblinger, D.; Zink, C.; Spencer, N. D.; Addadi, L.; Geiger, B. Effects of Surface Microtopography on the Assembly of the Osteoclast Resorption Apparatus. J. Royal Soc. Interface 2011, 9, 9568-9575.

(43) Tonna, E. H3-Histidine and H3-Thymidine Autoradiographic Studies of the Possibility of Osteoclast Aging. Lab. Invest. 1966, 15, $435-448$

(44) Hao, J.; Kuroda, S.; Ohya, K.; Bartakova, S.; Aoki, H.; Kasugai, S. Enhanced Osteoblast and Osteoclast Responses to a Thin Film Sputtered Hydroxyapatite Coating. J. Mater. Sci.: Mater. Med. 2011, 22, 1489-1499.

(45) Bang, S.-M.; Moon, H.-J.; Kwon, Y.-D.; Yoo, J.-Y.; Pae, A.; Kwon, I. K. Osteoblastic and Osteoclastic Differentiation on Sla and Hydrophilic Modified Sla Titanium Surfaces. Clin. Oral Implants Res. 2014, 25, 831-837.

(46) Nakamura, M.; Hentunen, T.; Salonen, J.; Nagai, A.; Yamashita, K. Characterization of Bone Mineral-Resembling Biomaterials for Optimizing Human Osteoclast Differentiation and Resorption. J. Biomed. Mater. Res., Part A 2013, 101, 3141-3151.

(47) Minkin, C.; Marinho, V. C. Role of the Osteoclast at the BoneImplant Interface. Adv. Dent. Res. 1999, 13, 49-56.

(48) Takemoto, M.; Fujibayashi, S.; Neo, M.; Suzuki, J.; Matsushita, T.; Kokubo, T.; Nakamura, T. Osteoinductive Porous Titanium Implants: Effect of Sodium Removal by Dilute Hcl Treatment. Biomaterials 2006, 27, 2682-2691.

(49) Oreffo, R. O. C.; Mundy, G. R.; Seyedin, S. M.; Bonewald, L. F. Activation of the Bone-Derived Latent Tgf Beta Complex by Isolated Osteoclasts. Biochem. Biophys. Res. Commun. 1989, 158, 817-823.

(50) Xian, L.; Wu, X.; Pang, L.; Lou, M.; Rosen, C. J.; Qiu, T.; Crane, J.; Frassica, F.; Zhang, L.; Rodriguez, J. P. Matrix Igf-1 Maintains Bone Mass by Activation of Mtor in Mesenchymal Stem Cells. Nat. Med. 2012, 18, 1095-1101.

(51) Lee, S.-H.; Rho, J.; Jeong, D.; Sul, J.-Y.; Kim, T.; Kim, N.; Kang, J.-S.; Miyamoto, T.; Suda, T.; Lee, S.-K. V-Atpase V0 Subunit D2Deficient Mice Exhibit Impaired Osteoclast Fusion and Increased Bone Formation. Nat. Med. 2006, 12, 1403-1409.

(52) Taranta, A.; Migliaccio, S.; Recchia, I.; Caniglia, M.; Luciani, M.; De Rossi, G.; Dionisi-Vici, C.; Pinto, R. M.; Francalanci, P.; Boldrini, R. Genotype-Phenotype Relationship in Human Atp6i- 
Dependent Autosomal Recessive Osteopetrosis. Am. J. Pathol. 2003, 162, 57-68.

(53) Karsdal, M. A.; Neutzsky-Wulff, A. V.; Dziegiel, M. H.; Christiansen, C.; Henriksen, K. Osteoclasts Secrete Non-Bone Derived Signals That Induce Bone Formation. Biochem. Biophys. Res. Commun. 2008, 366, 483-488.

(54) Pederson, L.; Ruan, M.; Westendorf, J. J.; Khosla, S.; Oursler, M. J. Regulation of Bone Formation by Osteoclasts Involves Wnt/ Bmp Signaling and the Chemokine Sphingosine-1-Phosphate. Proc. Natl. Acad. Sci. U.S.A. 2008, 105, 20764-20769.

(55) Ryu, J.; Kim, H. J.; Chang, E.-J.; Huang, H.; Banno, Y.; Kim, H.-H. Sphingosine 1-Phosphate as a Regulator of Osteoclast Differentiation and Osteoclast-Osteoblast Coupling. EMBO J. 2006, 25, 5840-5851.

(56) Takeshita, S.; Fumoto, T.; Matsuoka, K.; Park, K.-a.; Aburatani, H.; Kato, S.; Ito, M.; Ikeda, K. Osteoclast-Secreted Cthrc1 in the Coupling of Bone Resorption to Formation. J. Clin. Invest. 2013, 123, 3914-3924.

(57) Matsunaga, T.; Inoue, H.; Kojo, T.; Hatano, K.; Tsujisawa, T.; Uchiyama, C.; Uchida, Y. Increase in the Potential of Osteoblasts to Support Bone Resorption by Osteoclasts in Vitro in Response to Roughness of Bone Surface. Calcif. Tissue Int. 1999, 65, 454-458.

(58) Lossdörfer, S.; Schwartz, Z.; Wang, L.; Lohmann, C. H.; Turner, J. D.; Wieland, M.; Cochran, D. L.; Boyan, B. D. Microrough Implant Surface Topographies Increase Osteogenesis by Reducing Osteoclast Formation and Activity. J. Biomed. Mater. Res., Part A 2004, 70, 361-369.

(59) Miron, R. J.; Zohdi, H.; Fujioka-Kobayashi, M.; Bosshardt, D. D. Giant Cells around Bone Biomaterials: Osteoclasts or MultiNucleated Giant Cells? Acta Biomater. 2016, 46, 15-28.

(60) Miron, R. J.; Bosshardt, D. D. Multinucleated Giant Cells: Good Guys or Bad Guys? Tissue Eng., Part B 2018, 24, 53-65.

(61) Thudium, C. S.; Moscatelli, I.; Flores, C.; Thomsen, J. S.; Brüel, A.; Gudmann, N. S.; Hauge, E.-M.; Karsdal, M. A.; Richter, J.; Henriksen, K. A Comparison of Osteoclast-Rich and Osteoclast-Poor Osteopetrosis in Adult Mice Sheds Light on the Role of the Osteoclast in Coupling Bone Resorption and Bone Formation. Calcif. Tissue Int. 2014, 95, 83-93.

(62) Henriksen, K.; Bollerslev, J.; Everts, V.; Karsdal, M. A. Osteoclast Activity and Subtypes as a Function of Physiology and Pathology-Implications for Future Treatments of Osteoporosis. Endocr. Rev. 2011, 32, 31-63. 